\title{
Mesenchymal Stem Cell/Multipotent Stromal Cell Augmentation of Wound Healing
}

\section{Lessons from the Physiology of Matrix and Hypoxia Support}

\author{
Kyle Sylakowski, ${ }^{* \dagger}$ Andrew Bradshaw, ${ }^{* \dagger \dagger}$ and Alan Wells ${ }^{* \dagger \dagger}$
}

From the Department of Pathology* and the McGowan Institute for Regenerative Medicine, ${ }^{\ddagger}$ University of Pittsburgh, Pittsburgh; and R\&D Service, ${ }^{\dagger}$ VA Pittsburgh Health System, Pittsburgh, Pennsylvania

Accepted for publication March 25, 2020.

Address correspondence to Alan Wells, M.D., D.M.Sc., Department of Pathology, University of Pittsburgh, Scaife 713, Pittsburgh, PA 15261. E-mail: wellsa@upmc. edu.

\begin{abstract}
Cutaneous wounds requiring tissue replacement are often challenging to treat and result in substantial economic burden. Many of the challenges inherent to therapy-mediated healing are due to comorbidities of disease and aging that render many wounds as chronic or nonhealing. Repeated failure to resolve chronic wounds compromises the reserve or functioning of localized reparative cells. Transplantation of mesenchymal stem cells/multipotent stromal cells (MSCs) has been proposed to augment the reparative capacity of resident cells within the wound bed to overcome stalled wound healing. However, MSCs face a variety of challenges within the wound micro-environment that curtail their survival after transplantation. MSCs are naturally pro-angiogenic and proreparative, and thus numerous techniques have been attempted to improve their survival and efficacy after transplantation, many with little impact. These setbacks have prompted researchers to re-examine the normal wound bed physiology, resulting in new approaches to MSC transplantation using extracellular matrix proteins and hypoxia preconditioning. These studies have also led to new insights on associated intracellular mechanisms, particularly autophagy, which play key roles in further regulating MSC survival and paracrine signaling. This review provides a brief overview of cutaneous wound healing with discussion on how extracellular matrix proteins and hypoxia can be utilized to improve MSC retention and therapeutic outcome. (Am J Pathol 2020, 190: 1370-1381; https://doi.org/10.1016/j.ajpath.2020.03.017)
\end{abstract}

The skin is one of the largest organs of the body and plays an integral role in maintaining homeostasis with the world around us through three critical functions: regulation, protection, and sensation. Damage to the skin can make it lose one or more of these functions and subsequently kick start the cutaneous wound healing machinery to restore balance. In most cases for patients who are young and healthy, there will be a successful wound resolution, leading to restoration of normal skin functions. However, patients who are older or experience an array of diseases, such as cardiovascular diseases, diabetes, or obesity, have significantly higher impairment in their wound healing machinery. Currently in the United States, 8.2 million Medicare beneficiaries require interventional wound treatments, costing approximately $\$ 100$ billion. $^{1}$ The number of these patients is expected to increase over the next decade because of increasing age of the population compounded by the increase in comorbidities of chronic diseases.

These challenges compromise the reserve or functioning of localized reparative cells, particularly in wounds that require new tissue formation. Mesenchymal stem cells, or multipotent stromal cells (MSCs), have been proposed as a treatment to augment the reparative capacity in patients needing a biological boost to their nonhealing wounds.

Supported by NIH/National Institute of General Medical Sciences grant GM069668 (A.W.) and GM063569 (A.W.) and NIH/National Institute of Biomedical Imaging and Bioengineering training grant EB001026 (K.S. and A.B.).

Disclosures: None declared. 
MSCs have become coveted for their pro-angiogenic and immune-modulatory properties in the regenerative medicine space. However, one major complication limiting the efficacy of MSCs is their poor survival rate after transplantation. This is due to the hostility of the wound microenvironment, with ischemia being one of the most frequent challenges to overcome. This has led to the development of various strategies to improve MSC survival and angiogenic efficacy to overcome the ischemic barrier. Although many of such approaches are pharmaceutical or genetic modifications to the MSCs themselves, several research groups have attempted to harness the physiological survival and promotion signals native to a healthy wound healing micro-environment. Herein, we will focus on MSC combinational strategies utilizing matrix proteins and hypoxia as natural influences from the wound bed to augment survival and efficacy when used as exogenous cells to promote near regenerative cutaneous wound healing.

\section{Cutaneous Wound Healing}

Surface wounds are broken down into two major categories, depending on the need for tissue regeneration during healing. Surgical wounds and simple cuts are restored by juxtaposing the open wound edges together and closed mainly by localized matrix remodeling. However, when wounds, such as burns, abrasions, and traumatic wounds, present with lost tissue, a complex cascade of events ensues (Figure 1). An initial hemostatic and inflammatory phase limits the exposure to the external environment and restores a sterile barrier. This is followed through a tissue replacement phase and to successful resolution (scar). However, if complications occur during the early stages of healing with setbacks such as an insufficient immune response or hindered angiogenesis, then the wound will often become chronic or nonhealing. Chronic wounds are defined as ulcers or open wounds that fail to resolve within 3 months and are further classified into three main subgroups: vascular ulcers, pressure ulcers, and diabetic ulcers. ${ }^{2,3}$ The severity of chronic wounds will be unique for each patient and will need to be treated with a multifaceted approach by the clinicians. If unable to achieve wound healing advancement, then patients run the risk of infection, further spreading of damaged tissue, and amputation. Because of the gravity of repercussions that nonhealing wounds present, this is the area where scientists and clinicians are most focused on implementing new biological therapies. In contrast to chronic wounds, abnormalities in the last two stages of wound healing can result in a wound with excessive scarring, such as keloid or hypertrophic scar. ${ }^{3-5}$ Although the wound appears epithelialized and healed, these scars are dysfunctional and have an underlying dermis that is disfigured and compromised. The improper healing in these scars results in wounds that close but are structurally weaker and prone to re-ulceration.
The complete wound healing cascade is triggered when an injury damages the dermal and subdermal layers of the skin. The subsequent wound healing response is an intricate orchestration of three overlapping phases of repair that encompass numerous cell types, signaling cascades, and micro-environment modifications to reach a successful resolution (Figure 1). There are three main phases of wound healing: the hemostasis/inflammation phase, the tissue replacement phase, and the resolution phase. The first phase of wound repair is the hemostasis/inflammation phase, where first point of concern is to stop the bleeding of ruptured blood vessels while subsequently preventing pathogenic infection. On injury, a clotting cascade is activated where blood circulating platelets and enzymatic converted fibrin come together to form a fibrin clot and provide an early provisional matrix over the wounded area. ${ }^{6}$ This fibrin clot is also biologically active, playing a major role in signaling a localized immune response through the recruitment of pro-inflammatory macrophages (M1) and leukocytes to prevent infection and clear the wound of cellular and extracellular debris.

The next phase of wound healing, the tissue replacement phase, begins with the in-migration of fibroblasts, endothelial cells, epidermal cells, and other progenitor cells into the wound bed to initiate the rebuilding of the injured tissue. Fibroblasts begin producing the collagen III-rich granulation tissue as a temporary supportive matrix to replace the absent extracellular matrix (ECM); endothelial cells begin to generate new blood vessels via angiogenesis; and the epidermal cells start to migrate underneath the scab to permanently seal the wound surface and restore the epidermis. At this point, the wound is considered sterile and a transition occurs where pro-inflammatory macrophages (M1) decline and are replaced by wound healing macrophages (M2). These M2 macrophages help to produce and modulate the granulated ECM and promote further vasculature repair.

The resolution phase is the final repair phase where the provisional wound bed is replaced with a mature ECM, the excess blood vessels are pruned by up to $90 \%,{ }^{7}$ and any residual proliferation and migration signals are terminated and replaced with stop signals, such as those acting through CXCR $3 .{ }^{8}$ The wound bed will start to contract as fibroblasts transdifferentiate into myofibroblast to reorganize and restructure the immature collagen III to mature collagen I, restoring tensile strength back to the skin. ${ }^{9}$ With every phase having a successful outcome, all that will remain will be a light scar to hint at what occurred.

\section{Mesenchymal Stem Cells in Wound Repair}

Mesenchymal stem cells, or MSCs, are characterized for their ability to self-renew, adhere to plastic, and differentiate into subsets of specialized cells particularly suited for the regeneration of mesenchymal tissues (adipocytes, 
osteoblasts, chondrocytes, and myoblasts). ${ }^{10-16}$ They are further defined by the International Society for Cell and Gene Therapy for expressing markers CD73, CD90, and CD105 while also lacking the expression of markers CD14, CD19, CD34, CD45, CD11b, CD79a, and human leukocyte antigen-DR. ${ }^{17}$ MSCs as a cellular therapy have shown clinical benefit in several disease situations, although this has related to the immunosuppressive activities rather than tissue generation. ${ }^{18,19}$ Unfortunately, this approach has proved disappointing in wound repair despite success in preclinical animal models as the stem cells are lost rapidly from the wound bed. A more recent area of success using MSCs has been for angiogenesis and improved healing among different tissue types. This is because of the variety of trophic factors that MSCs have been found to secrete in context to their micro-environment. ${ }^{18,19}$ Still, to achieve any significant benefit, the MSCs must remain for the time scale of the wound healing phase, which runs in weeks.

The use of MSCs to treat dysfunctional cutaneous wounds is considered particularly promising for several reasons. First, the immune-tolerant site of the skin can accommodate extended persistence of allogeneic cells. Second, skin being an externally accessible organ, repeated applications can be achieved noninvasively. In addition, the proreparative secretome of MSCs can be utilized in different phases of the wound healing process (Figure 1). This is an

\section{Hemostasis/} Inflammation
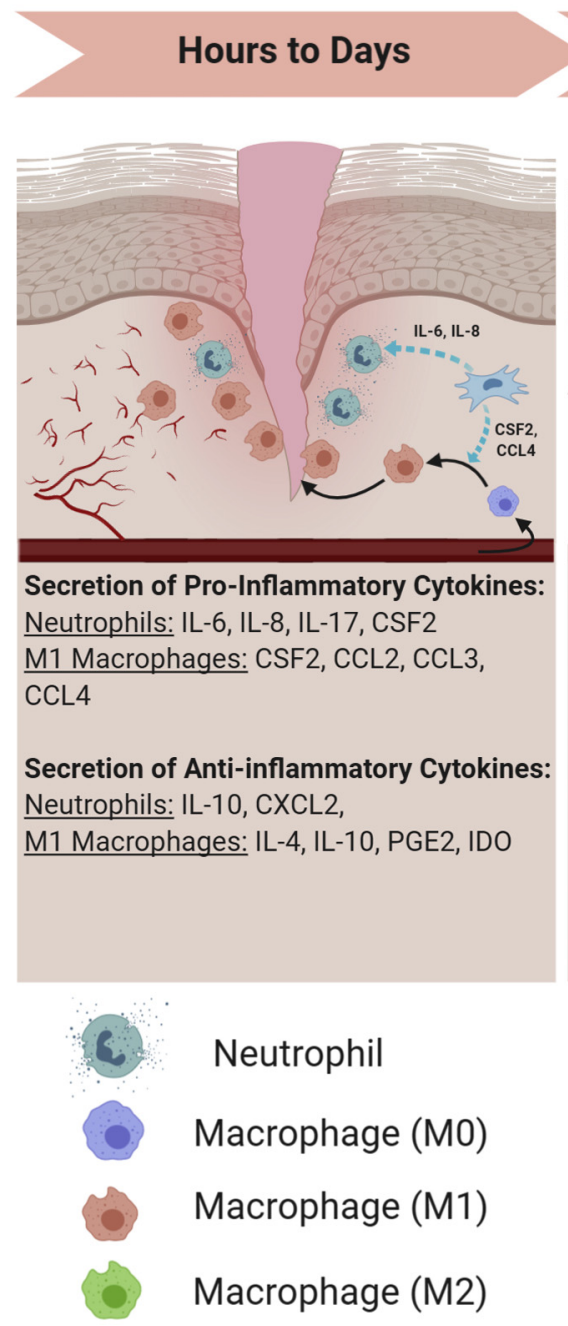

\section{Tissue Replacement}

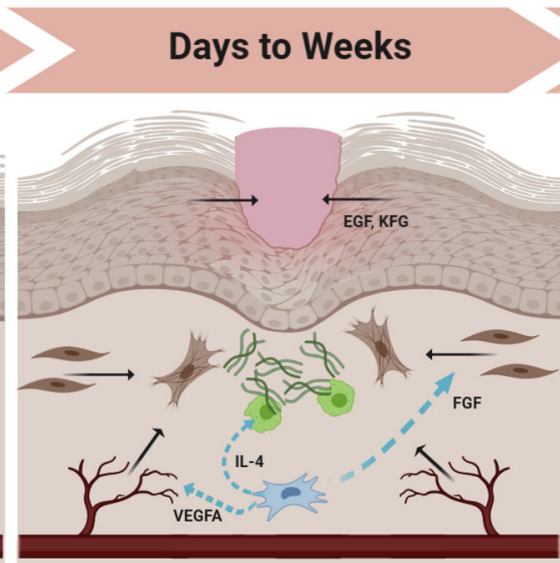

Epidermal Cell Recruitment and Migraton: Epithelial Cells: EGF, KGF, HGF Dermal Proliferation and Migration: Fibroblasts: FGF Progenitor/Stem Cells: CXCL12, IGF-1 Angiogenesis: Endothelial Cells: VEGFA, ANG, IL-8, CCL2, PDGF-BB

Macrophage Recruitment/Conversion: M2 Macrophage: IL-4, IL-13, IL-10, TGF- $\beta$ Matrix Remodeling: MMPs and TIMPs

\section{Resolution}

\section{Weeks to Years}

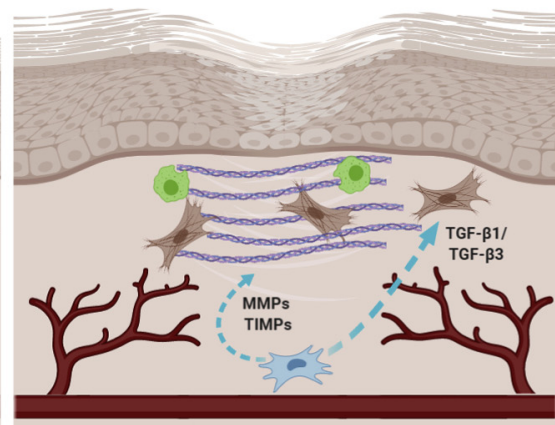

Matrix Remodeling:

MMP2, MMP9, MMP14, TIMP1, TIMP2

Regulation of Collagen Deposition:

Myofibroblasts: TGF- $\beta 1$ and TGF- $\beta 3$ ratio


Fibroblast
Myofibroblast
Mesenchymal Stem Cell

Collagen 1

Collagen 3

Broken Blood Vessel

Figure 1 Roles for mesenchymal stem cells (MSCs) during cutaneous wound healing. The hemostasis/inflammation phase is heavily reliant on the proinflammatory machinery for sterilizing the wound and clearing damaged extracellular matrix debris. MSCs help to orchestrate a healthy initial immune response that eventually will turn over into a proreparative response. On entering the tissue replacement phase, MSCs produce various growth factors and chemokines to initiate a massive arrival of tissue resident cell types that will begin to rebuild within the wound bed. The resolution phase is the final phase of wound healing where MSCs help to orchestrate final modifications to the extracellular matrix, vasculature, and resident cells. BioRender (Toronto, 0N, Canada) was used to design and generate the figure. CCL, chemokine (C-C motif) ligand; EGF, epidermal growth factor; FGF, fibroblast growth factor; PDGF-BB, plateletderived growth factor-BB; TGF- $\beta$, transforming growth factor- $\beta$; VEGF, vascular endothelial growth factor. 
advantage over direct application of growth factors or other molecules as the wound bed consists of asynchronous areas of healing, where MSCs are environmentally adaptive and can produce an appropriate response for the transitioning phases of the wound bed to recreate the physiological situation. In a normal skin wound, endogenous subsets of hair follicle MSCs called dermal sheath cells help to repair/ replace the injured dermal tissue. MSCs from the subcutaneous fat tissue and blood supply help regulate early and mid-phase inflammation while also helping to restore dermal tissue. ${ }^{20,21}$

On infiltrating the wound, MSCs begin to secrete proinflammatory cytokines [CSF2, IL-6, IL-8, and chemokine (C-C motif) ligands 2 and 3], recruiting neutrophils and M1 converted macrophages to help degrade damaged tissue ${ }^{21,22}$ (in the hemostasis/inflammation phase), but also regulating the overall inflammatory response by limiting the number of activated $\mathrm{T}$ cells, neutrophils, and macrophages. ${ }^{21,23-25} \mathrm{On}$ transition into the tissue replacement phase, MSCs focus on secreting cytokines directed to the proliferation and migration of epithelial cells for re-epithelialization [epidermal growth factor, keratinocyte growth factor (KGF), hepatocyte growth factor (HGF)], directing new endothelial cells for angiogenesis [vascular endothelial growth factor (VEGF) A, angiopoietin 1 (ANGPT1), and platelet-derived growth factor-BB], and stimulating fibroblasts to produced matrix proteins that later transition to a mature provisional matrix (fibroblast growth factor). ${ }^{21,22,26,27}$ MSCs also direct the polarization of monocytes into proreparative M2 macrophages (IL-4, IL-13, IL-10, and transforming growth factor$\beta)$ to help clear remaining cellular debris and modify temporary matrix. ${ }^{21,24}$ In the resolution phase, MSCs continue to regulate the matrix through secretion of matrix metalloproteinases (MMPs) and tissue inhibitor of metalloproteinases (TIMPs), while also regulating the amount of collagen disposition through balancing transforming growth factor- $\beta 1$ and transforming growth factor- $\beta 3$ to prevent hypertrophic scarring. ${ }^{27}$ These vital guiding functions are unique to MSCs because they express low levels of major histocompatibility complex class II and lack the major histocompatibility complex costimulatory molecules that are essential for immune cell activation (CD40, CD40L, CD80, and CD86), allowing for their use in allogeneic transplant with a low rate of rejection. ${ }^{28}$

\section{Hostile Wound Micro-Environment Challenges MSC Survival}

Despite the potential benefits MSCs possess for regenerative therapies, their use must first overcome pro-apoptotic stressors within wounded tissue. ${ }^{29}$ Younger and healthier patients have wound healing machinery and endogenous MSCs that work well enough to overcome these healing challenges. However, in patients who are older and experience diseases, such as those with type 2 diabetes or metabolic syndrome, their MSCs become more susceptible to apoptosis, increased levels of reactive oxygen species accumulation, and increased mitochondrial deterioration. This renders their MSCs too dysfunctional ${ }^{30}$ to be able to overcome the stress of the wound bed, ultimately limiting their effectiveness in the wound healing process. This is where the reliance on exogenous MSC therapies from healthier and younger donors has come to light. However, the lack of survival of exogenously applied MSC in wounds has still been well documented in a variety of tissues, particularly the heart, ${ }^{31}$ brain, ${ }^{32}$ and kidney. ${ }^{33}$ For the heart, there was a survival rate of $5 \%$ within a 2 -week period after myocardial infarction treatment, ${ }^{31}$ and only a $1 \%$ survival rate 1 hour after injection into an ischemic kidney model. ${ }^{33}$ Even when using an immunodeficient mouse model, Toma et $\mathrm{al}^{34}$ report having $<0.44 \%$ survival of MSCs 4 days after myocardial infarction. Even skin, where immune tolerance should present less barriers to MSC, is devoid of implanted MSCs within a week. ${ }^{35}$

The disparagingly low survival rates likely result from a variety of factors within the harsh wound micro-environment, such as the absence of trophic factors, a heightened inflammatory response, and an impaired vasculature. ${ }^{36,37}$ These factors induce MSC death through a variety of mechanisms, including anoikis, ischemic reactive oxygen species, loss of growth factors, or increased signaling from death cytokines ${ }^{38}$ (Figure 2). Previous studies have shown that MSCs are susceptible to reactive oxygen species and pro-inflammatory death, inducing signaling complexes that occur within the wound bed. ${ }^{39}$ Also, MSCs are metabolically glycolytic, ${ }^{40}$ so that nutrient deprivation within the wound bed is also potentially detrimental to MSC survival. ${ }^{41,42}$ Recent evidence regarding hypoxic/ischemic stress showed that severe hypoxia $\left(<1 \% \mathrm{O}_{2}\right)$ drives mitochondrial dysfunction, resulting in apoptosis or necrosis. ${ }^{41,43}$ Despite numerous approaches to limit MSC death after transplantation, whether through growth factor or drug preconditioning or genetic modifications, ${ }^{44}$ most efforts have had a limited impact on overall MSC survival. The death of the exogenously applied MSCs is in counterpoint to the survival of the endogenous MSCs. These lessons from the physiological adaptations and survival mechanisms of successful resolving wounds can be used to develop prosurvival therapies for elderly individuals and those with dysfunctional MSCs or comorbidities.

\section{MSC Delivery Strategies for Improved Survival and Efficacy}

Cutaneous wounds requiring interventional treatment options are often complex and require a multidisciplinary approach both at the macro level and micro levels of care. Macro levels of care would be considered more general hospital methods of treatment, such as site off loading, compression bandages, and fluid control. Micro level of care would be anything using a biological treatment focused on 


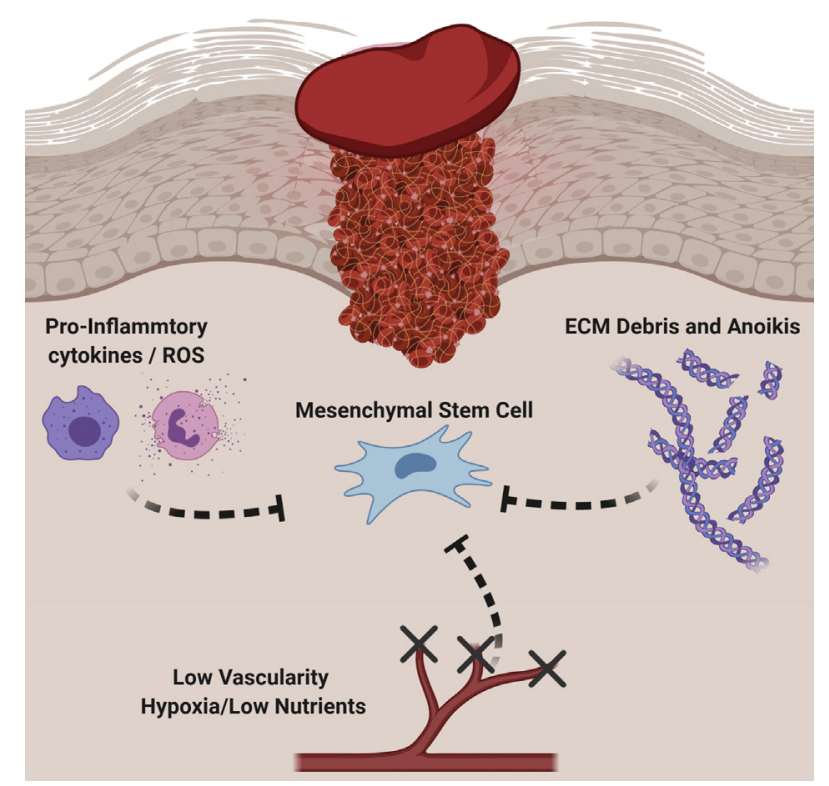

Figure 2 Mesenchymal stem cells (MSCs) face a steep challenge when they enter the wound micro-environment. Several factors are in play that will limit their survival and therapeutic capacity. The innate immune response is responsible for destroying any foreign pathogens, but their methods of destruction can be nondiscriminatory through their release of pro-inflammatory cytokines and reactive oxygen species (ROS). The insult will also cause tissue damage, leaving behind extracellular matrix (ECM) debris that can result in a lack of support to cells in the area, resulting in cell death via anoikis. Injury to the wound bed can also cause blood vessels to be severed, subsequently resulting in all damaged vasculature to be blocked off. This will cause the wound micro-environment to become ischemic, leaving all cells to be with little to no oxygen or nutrients available. BioRender (Toronto, ON, Canada) was used to design and generate the figure.

improving the wound at the micro-environment level. There are many elements clinicians and scientists must consider when utilizing and developing MSC therapeutic strategies. Cell source is often a major consideration because there are multiple types of MSC-derived lineages that can behave differently under varying circumstances. In addition, there are some sources of MSCs that are easier to extract and with greater numbers than with other locations of the body. The two most common sources of MSCs for clinical use are derived from either the bone marrow, where $0.001 \%$ to $0.002 \%$ of cells harvested are MSCs, or adipose tissue, where MSCs are estimated to make up $1 \%$ of the total cell population. ${ }^{45}$ Cell administration strategy is another key factor and will change depending on the patients' wound severity and type. But, overall, the main goal of improving MSC survival percentages after transplantation remains a focal point for enhancing MSC efficacy in wound healing. Herein, we focus on two of the most promising approaches for improving MSC retention: extracellular matrix construct and hypoxic preconditioning. Both of these methods rely on the impact of environmental cues on the MSCs to influence their behaviors rather than artificial micromanipulations, such as gene editing techniques or pharmaceutical drugs.
The overarching idea for these MSC delivery strategies is to utilize components native to the wound healing environment/repair mechanisms to reduce extraneous stress that other, more artificial methods may add to an already dysfunctional system. Both extracellular matrix compositions and hypoxia/ischemia are central aspects of the inflammatory and tissue replacement phases of wound healing, and thus can be considered physiological mimics.

\section{Matrix Components for MSC Delivery}

The extracellular matrix is now appreciated as being just as important as the cells themselves during healing. ${ }^{3,40}$ The ECM is not just the major support structure of each tissue, but the tension combined with bioactive moieties within the ECM largely influences how cells within the tissue function and behave. The ECM's biochemical makeup, rigidity, and shape are being exploited to manipulate tissue replacement and wound healing across many applications. Using ECM constructs that either resemble or impute the native matrix environment could help in promoting more physiological healing.

Collagen I is the most abundant ECM protein of the skin and is usually preferred for shovel-ready scaffolds as it is biocompatible, is biodegradable, and can form highly organized two- and three-dimensional network-like structures that allow it to incorporate a wide variety of biological components, ranging from growth factors to matrix proteins to cells. Collagen I has been shown to improve MSC proliferation potential and prevent MSC anoikis through the binding of integrin receptors $\alpha 2 / \beta 1$ and $\alpha 11 / \beta 1{ }^{46,47}$ Collagen I is also able to increase osteogenic differentiation through higher cell adhesion and enhanced activation of RHO-A in vitro. ${ }^{46}$ Another structural collagen, collagen VI, has been used to enhance MSC proliferation and stemness for cartilage repair, while also displaying prosurvival capabilities in fibroblasts. ${ }^{48,49}$ Thus, encapsulating MSCs within structural collagen may augment the delivery of viable MSCs. However, collagen I signals wound maturation and resolution, and thus would be suppressive during the early tissue replacement phase of healing; more important, collagen I-dominant matrices limit angiogenesis. ${ }^{40}$

A more immature matrix is likely needed to promote regenerative healing. ${ }^{50}$ The matricellular protein tenascin-C (TNC) possesses epidermal growth factor receptor-like repeats that can interact with the epidermal growth factor receptor (EGFR) on MSCs in a low-affinity/high-avidity interaction; this restricts EGFR activation to the plasma membrane. ${ }^{29,51}$ The prolonged sequestration of EGFR enhances prosurvival signals, via low-level tonic extracellular signal-regulated kinase and AKT signaling. ${ }^{37,52}$ The addition of TNC to MSCs provides for survival of the transplanted cells for up to a month. ${ }^{26,35}$ Given that TNC is found naturally during the early phases of wound healing, coupling it with MSCs as a therapeutic delivery system is expected to not only provide for enhanced survival but also 
improve healing. ${ }^{26,35}$ Laminin $\mathrm{V}$ is another promising immature matrix protein that contains cryptic EGFR matrikines. ${ }^{52}$ Combinational strategies using laminin with MSCs have shown to enhance overall survival and improve wound healing outcomes through up-regulated angiogenic capacity in diabetic rats and other models. ${ }^{53,54}$ The wound response matrix component fibronectin is another top candidate for MSC delivery. MSC survival was enhanced in a rat hind limb model when encapsulated in agarose capsules containing fibronectin and fibrinogen prosurvival signals,${ }^{55}$ in addition to increasing proangiogenic capacity of MSCs in vitro. ${ }^{54}$

In addition to improving MSC survival and paracrine signaling, many of these immature matrices also promote the migration of cells around the wound bed. Tenascin-C, laminin, fibronectin, and thrombospondin all play vital roles in orchestrating the movement of distal proliferating resident cells to the center of the wound bed, mainly through integrin binding mechanisms. ${ }^{56-58}$ These properties add another facet to the overall capability of these MSC-ECM combinational therapies. The immature matrices improve the survival and growth factor secretion of MSCs, which then promotes enhanced proliferation of the resident tissue cells, which, in turn, are able to migrate into the wound quicker because of the aforementioned present immature matrix. Further adding to this enhanced cycle of repair, MSCs have also shown that they can deposit ECM proteins, such as fibronectin, tenascin-C, thrombospondin, and others. ${ }^{59}$ All together, these matricellular proteins are highly regulated in adult tissues with restricted expression to areas of active remodeling, such as in wound healing. ${ }^{60}$ Thus, by following the lessons from physiological healing, novel approaches can be designed employing the same matricellular proteins.

\section{Hypoxic Preconditioning for MSC Delivery}

Many MSCs reside in areas of low oxygen tension, from 3\% to $9 \%$, depending on the tissue source. ${ }^{61,62}$ Hypoxia within the stem cell niche is thought to maintain self-renewal, proliferation, and migration, and ultimately appears to augment their therapeutic potential. ${ }^{63-65}$ Taking these cues from physiology, this has led to experiments in which MSCs are grown at similarly low levels of oxygen to condition or acclimate them before wound implantation. However, when oxygen levels reach extreme levels of hypoxia $\left(<1.5 \% \mathrm{O}_{2}\right)$, such as within a wound bed, MSCs can become overstressed and undergo apoptosis. ${ }^{64}$ Further studies have shown that MSCs are innately glycolytic, ${ }^{40}$ and it is the combination of low oxygen tension and nutrient deprivation within the ischemic wound environment that severely challenges the cells. MSCs exposed to near-anoxic conditions $\left(0.1 \% \mathrm{O}_{2}\right)$ for as little as 3 days were shown to rapidly consume all internal glucose energy reserves, resulting in poor survival after implantation. ${ }^{66}$
With both oxygen and nutrients being vital for MSC survival after implantation, researchers are focused on generating delivery methods that improve at least one of these bioenergetic challenges. The biggest and potentially easiest way to enhance MSC survival is to precondition MSCs in hypoxic conditions $\left(1 \%\right.$ to $\left.4 \% \mathrm{O}_{2}\right)$ for 24 to 48 hours before implantation. MSCs pre-exposed to these hypoxic conditions respond by up-regulating the hypoxiainducible factor $1 \alpha$ (HIF- $1 \alpha) .{ }^{67} \mathrm{HIF}-1 \alpha$ is a major regulator and response factor to oxygen tension within the cell, in which it will be post-translationally modified and marked for degradation under normal oxygen conditions. During hypoxia, HIF- $1 \alpha$ escapes degradation and translocates to the nucleus, where it will then activate hypoxia response elements. Many of the genes that hypoxia response elements target are involved in promoting angiogenesis (ie, VEGF), promoting survival (Bcl-2 and AKT), or regulating metabolism via increasing glycolytic potential (ie, glucose transporter 1, lactate dehydrogenase A, and phosphoglycerate kinase 1). ${ }^{68,69}$ The increase in glycolytic genes translates to increased MSC consumption of glucose and production of lactate in an oxygen-dependent manner. ${ }^{64,70}$ Further analysis of hypoxia response element target genes has also found active mitochondrial repressors, such as pyruvate dehydrogenase kinase 1 , an inhibitor of pyruvate entrance into the tricarboxcylic acid (TCA) cycle. Pyruvate dehydrogenase kinase 1 inhibition of pyruvate effectively reduces mitochondrial oxygen consumption and thus allows cells to preserve their intracellular oxygen concentrations. ${ }^{71}$ MSCs exposed to hypoxic conditions also utilize HIF- $1 \alpha$ to activate the AKT signaling pathway to enhance survival and proliferation. ${ }^{72}$ However, when HIF- $1 \alpha$ is knocked down, compensatory activation of the p53 pathway occurs, resulting in a higher induction of apoptosis. ${ }^{69}$ These manipulations appear to marginally improve the survival of the transplanted MSCs. ${ }^{70,73}$ Thus, the next steps would be a combination of hypoxic preconditioning with delivery within matricellular components, such as TNC.

\section{Autophagy May Be a Key Mechanism for Survival and Efficacy in MSC Combinational Therapies}

On further investigation into the effects of hypoxic preconditioning on MSCs, up-regulated HIF-1 $\alpha$ was shown to promote the initiation of macroautophagy. ${ }^{74}$ Macroautophagy (hereafter, autophagy), is an evolutionarily conserved self-eating catabolic process that targets cellular components for degradation through the formation of double membraned organelles called autophagosomes that later fuse with lysosomes. ${ }^{75}$ Under normal circumstances, autophagy is a highly selective process used to maintain cellular homeostasis by degrading the buildup of aggregate-prone proteins and dysfunctional organelles. ${ }^{76}$ However, when cells are exposed to environmental stressors, such as nutrient deprivation or hypoxia, autophagy becomes a nonselective process 


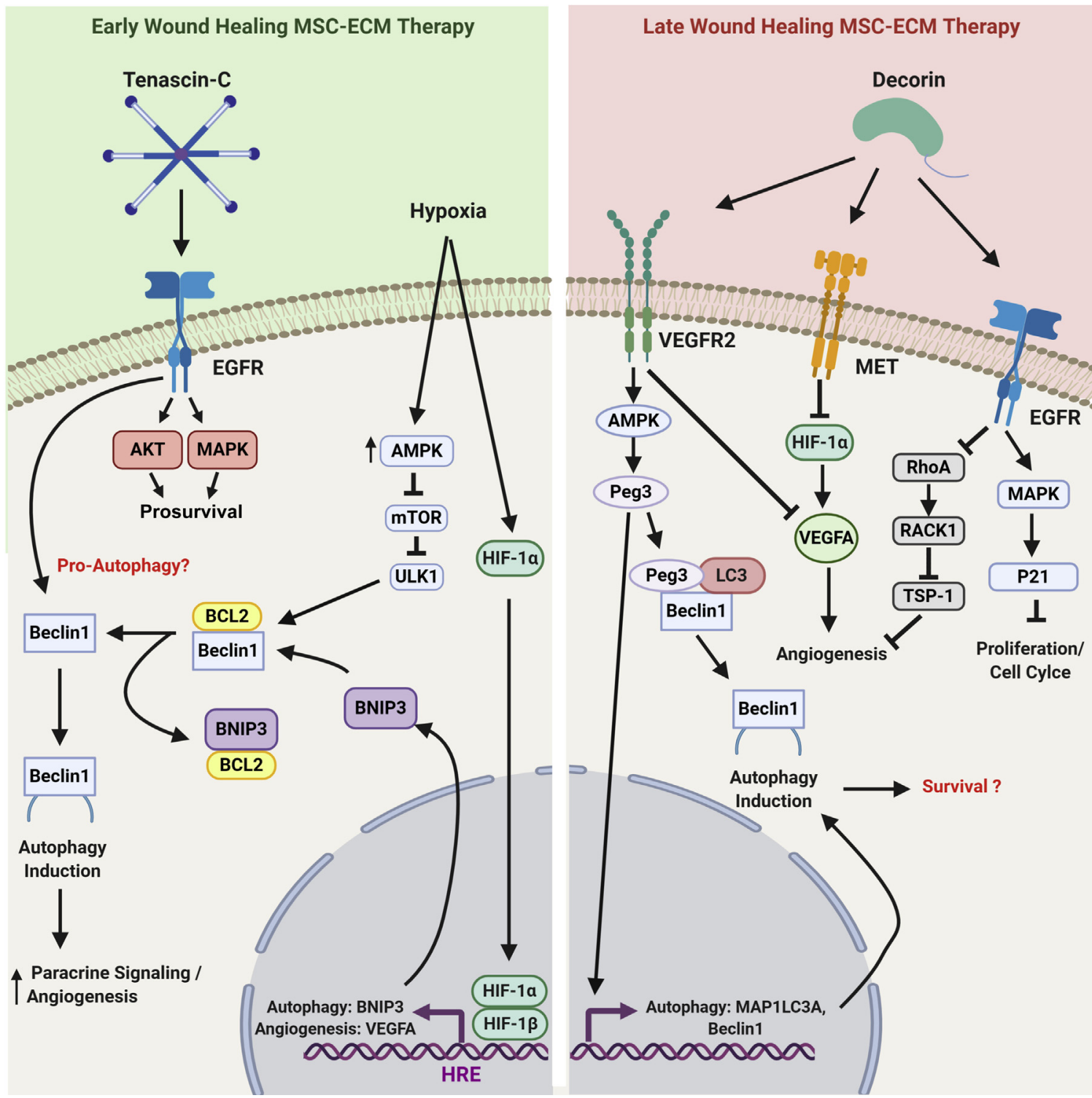

Figure 3 Mesenchymal stem cell (MSC)-extracellular matrix (ECM) combinational strategies need to be customized, depending on the target stage of wound healing. The interventions would be expected to have different influences on MSC performance based on the milieu. Left panel: An MSC-ECM combinational therapy targeting the inflammatory and tissue replacement phases of wound healing, such as in treating a chronic ulcer. Using a matrix protein that is naturally occurring during the early phases of wound repair, tenascin-C (TNC), will provide prosurvival signals through continual extracellular signal-regulated kinase and AKT signaling cascades. In conjunction with the TNC survival signals, the MSCs will be exposed to hypoxia/ischemia. This will jump start the hypoxia-inducible factor-1 $\alpha$ (HIF-1 $\alpha$ ) signaling complex and result in an up-regulated autophagic (BNIP3) and pro-angiogenic response [hypoxia response elements (HREs), such as vascular endothelial growth factor (VEGF)-A]. TNC is also linked to the induction of Beclin1 and has been postulated as an influencer of autophagic expression. With the addition of the autophagic machinery, the MSCs can utilize its output of nutrients/building blocks (amino acids, nucleic acids, fats, and sugars) to further enhance its pro-angiogenic signaling properties. Right panel: An MSC-ECM combinational therapy that would be used to target the resolution phase of wound healing in hopes of stopping excess scar formation. Decorin is a naturally occurring matrix protein during the resolution phase and works as a major stop signal for all of the prowound healing systems that were at play in the early phases of repair. It binds to various growth factors and receptors, where it signals the surrounding cells to stop proliferating [epidermal growth factor (EGF)] and stop producing angiogenic factors [VEGF receptor 2 (VEGFR2), MET, and EGF receptor (EGFR)]. However, Decorin is well known for inducing a robust induction of autophagy (VEGFR2) without a clear understanding of how that affects the physiology of the cells moving forward. BioRender (Toronto, ON, Canada) was used to design and generate the figures. MAPK, mitogen-activated protein kinase.

indiscriminately sequestering large amounts of cytosolic cargo for degradation in the attempt to prolong survival. ${ }^{77}$ The activation of nonselective autophagy quickly becomes a balancing act within a cell, as autophagy can play a role in both cell survival and apoptosis. ${ }^{78}$ In addition, autophagy and apoptosis mutually inhibit each other until an undefined sensitivity threshold is reached and one process overtakes the other, subsequently resulting in either adaptation of the cell to the stress or stress-induced death. ${ }^{43,79}$ It is through these threshold boundaries where MSCs can be further fine-tuned to escalate their efficacy as cell therapies. 


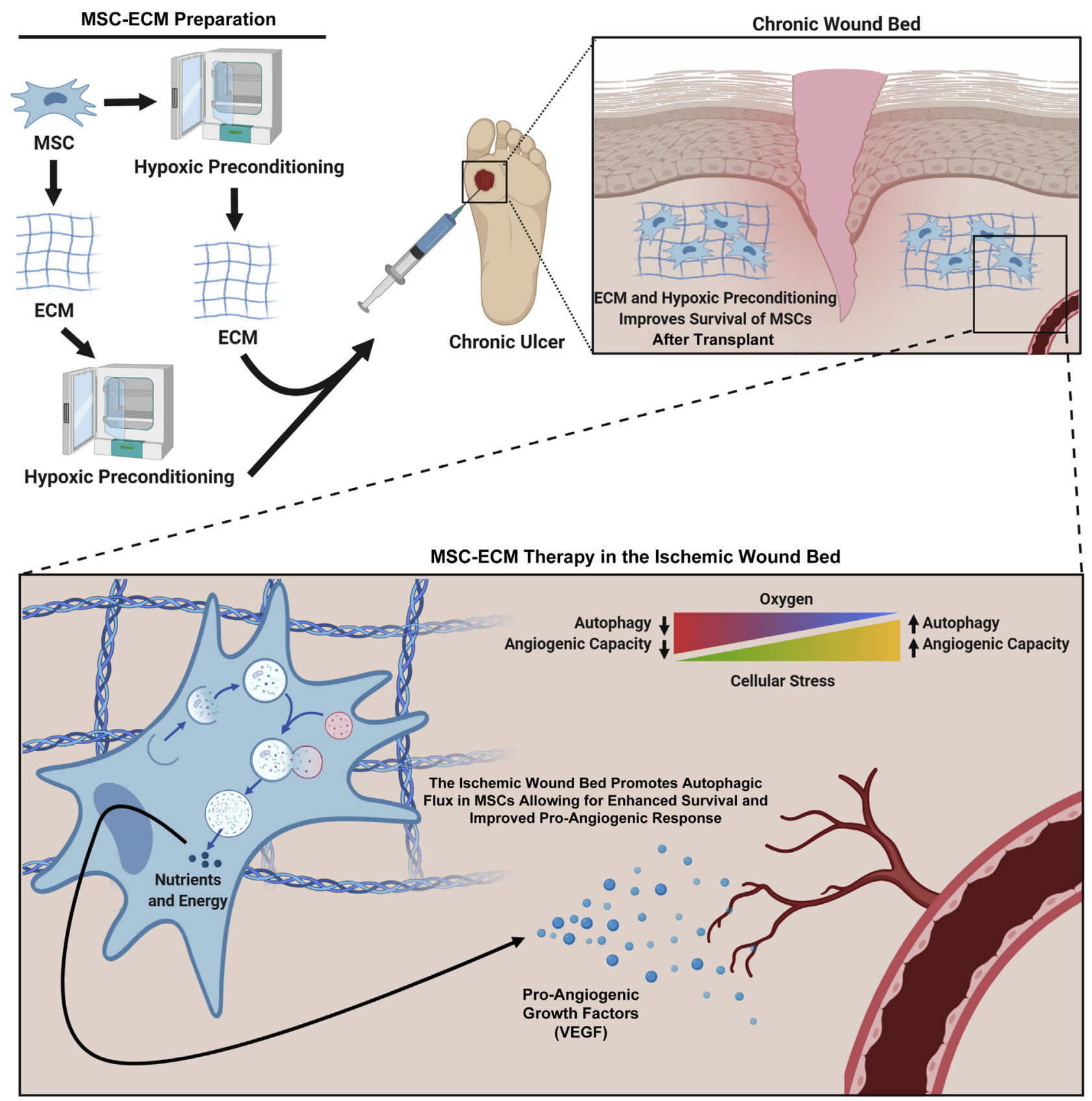

Figure 4 Schematic of combinational mesenchymal stem cell (MSC) therapies utilizing matrix and hypoxia to treat a chronic wound. This is envisioned as future development. The first step in the process would be to prepare the extracellular matrix (ECM) constructs and MSCs for delivery. The type of skin wound and the build of the ECM scaffold will determine whether MSCs need to be combined with the ECM before or after hypoxic preconditioning. Once the MSC-ECM sample has been prepared, it can be delivered to the wound. The combination of the ECM construct support and the hypoxic preconditioning will help the MSCs overcome the initial shock of the ischemic wound bed, allowing them to acclimate better to newly encountered cellular stressors. The ischemic environment will promote additional intracellular survival machinery, such as autophagy, allowing the MSCs to gain additional nutrients and energy for longer survival. Once the MSCs have stabilized, they will respond to the ischemic environment by utilizing the autophagy machinery and produce pro-angiogenic growth factors, such as vascular endothelial growth factor (VEGF), to promote angiogenesis. The restoration of new vessels will help enrich the surrounding resident cells and ultimately progress the wound forward in the healing process. BioRender (Toronto, ON, Canada) was used to design and generate the figure.

MSC performance and function are tied to its ability to regulate autophagy. ${ }^{80}$ To date, basal autophagy levels have been shown to be higher in MSC populations compared with differentiated cell types. ${ }^{81}$ Additional studies suggest that autophagy is required for conservation of MSC stemness and self-renewal capabilities. ${ }^{82}$ Even more interesting is that non-stressed MSCs accumulate arrested autophagosome, and their autophagic manipulation significantly alters the balance between renewal and differentiation. ${ }^{83}$ MSCdirected differentiation dynamics will change when autophagy is manipulated as early as 3 hours after induction into adipogenic and osteogenic lineages. ${ }^{83,84}$ This is attributable 
to the MSCs quickly consuming the arrested autophagosomes that were present before induction; and by speeding up or slowing down the autophagy process, this will either increase or decrease the overall differentiation efficiency outcomes. ${ }^{83}$

MSC paracrine function has also been linked to autophagic manipulation. This has been reported mostly in conjunction with hypoxic preconditioning. As MSCs experience hypoxia, HIF-1 $\alpha$ becomes up-regulated and allowed to translocate into the nucleus, and it initiates the production of BNIP3/BNIP3L, a set of pro-autophagy proteins that interact with the Bcl-2 sequestration complex, allowing Beclin-1 to dissociate away and initiate the autophagic cascade. ${ }^{85}$ In addition to BNIP3, hypoxia also positively influences autophagy induction through regulating the expression of MAP1LC3 $\beta$ and ATG5 via PERK-unfolded protein response (UPR) pathway, as well as activating the AMP-activated protein kinase (AMPK) pathway. ${ }^{86}$ This upregulation in autophagic flux has been linked to increased expression of VEGF secretion through direct phosphorylation of extracellular signal-regulated kinase. ${ }^{87}$ The combination of pretreating MSCs with hypoxia could enhance the therapeutic potential of MSCs even further, especially by promoting angiogenesis. ${ }^{88}$ In treating post-myocardial infarction hearts, MSCs that underwent hypoxic preconditioning exhibited higher rates of autophagic flux, resulting in higher retention in cell number and a significant reduction in scar formation within the infarct wall. ${ }^{89}$

The strong connection of autophagy to cellular processes, such as angiogenesis, has launched the cancer treatment and stem cell therapy fields to find additional upstream activation/regulation inputs. This has resulted in a large emphasis on the ECM as a point of interest for such studies. ${ }^{90}$ The most recent studies have focused on proteoglycans, primarily the small leucine-rich proteoglycan called Decorin. $^{91,92}$ Decorin becomes up-regulated within the resolution phase of wound healing, where mature matrix, such as collagen-I, becomes present again during this final stage of healing. Decorin is considered an important shutoff switch for the proreparative mechanism of the tissue replacement phase, as it binds to growth factor receptors, such as EGFR, VEGF receptor 2, and MET, and limits the occurrence of hypertrophic scarring through preventing excessive repair. On binding these receptors, Decorin will induce autophagy and mitophagy responses within the cell. ${ }^{91,93}$ However, these actions have been shown in differentiated cells and not yet in stem cells. It is also unclear which physiological roles Decorin-induced autophagy has in certain cell types. ${ }^{91}$ When postulating what this could mean for MSCs, Decorin could be helping to reset the stem cell niche back to its dormant state by down-regulating the proliferation and angiogenic signals once needed for early wound repair. And like other cells that remain in a quiescent state for long periods of time, they rely heavily on autophagy to clear out unwanted intracellular debris, unlike higher proliferating cell types.
For early wound healing situations, the immature matricellular TNC has been linked to prompt Beclin-1 induction, ${ }^{94}$ which could possibly induce autophagic flux. This would either be a second mode of survival mechanism (in addition to the tonic AKT and extracellular signal-regulated kinase signaling) or an additional driver in producing the secretome for tissue replacement. TNC is also known as being an anti-adhesive matrikine, allowing for the migration and ECM detachment of cells during development and wound repair. ${ }^{94}$ Loss of ECM attachment has been shown to induce autophagy to promote a survival mechanism against short-term anoikis. ${ }^{95}$ Thus, the anti-adhesive nature of TNC along with other ECM anti-adhesive glycoproteins could render a secondary method of action for inducing autophagy survival mechanism in MSC-ECM transplantation strategies. For most of these anti-adhesive ECM proteins to be properly combined with MSC delivery strategies in two or three dimensions, collagen I is often used as a structural tether to keep everything together. However, there is evidence in vitro that collagen I actively down-regulates autophagy, ${ }^{96}$ suggesting that further research will be needed to fine-tune the ratio of matrix protein delivery strategies, depending on the required outcome. This concept is even more important when considering what phase of wound healing is needing to be targeted. A prosurvival ECM protein, like TNC, is useful during the inflammation and tissue replacement phases of repair to help restore delayed wound healing; and Decorin is most useful during the resolution phase of wound healing to stop the proreparative system from overproducing and generating unwanted scarring (Figure 3). Altogether, both environmental stimuli of hypoxic preconditioning and matrix proteins are linked to autophagy in various ways, and further investigation into how to fine-tune their inputs in conjunction with autophagic flux could prove to be a key insight into optimizing MSC therapeutic approaches. This is especially true in essential processes, such as angiogenesis, where increased autophagic flux has been linked to up-regulating angiogenesis through increased production of VEGFA in cutaneous wound repair. ${ }^{87}$

\section{Conclusions and Future Directions}

MSCs have numerous attributes that make them attractive for facilitating healing in dysfunctional and nonfunctional wound beds. Their use as adaptive paracrine and matrixproducing factories is desirable in that eventually rejected allogeneic cells could still promote near-regenerative healing. However, MSCs face many challenges after transplantation into the wound bed. Overcoming these challenges is necessary to demonstrate their efficacy in treating injured tissue (Figure 4). We propose that successful use of MSCs in promoting more regenerative healing or completing stalled chronic wounds will ensue from learning from the physiology. 
Using preconditioning techniques and matrix support strategies to target autophagic responses could be a key in improving MSC survival. ${ }^{80}$ MSCs are inherently glycolytic $^{40}$ and will rapidly use internal energy reserves ${ }^{66}$ when subjected to extreme environments, such as ischemia in wound bed. Manipulating how MSCs utilize autophagic flux could help maintain internal energy stores for longer periods, leading to larger cell retention. Matricellular component-containing delivery vehicles not only impact autophagy and metabolic programming but also directly trigger survival pathways, further improving the persistence of transplanted MSCs. This increase in survival fraction would allow for the MSCs to exert trophic influences on angiogenesis and tissue replacement for the weeks-long period needed to transition through the phases to wound resolution.

\section{Acknowledgments}

We thank the Wells Lab and the laboratory of Partha Roy (University of Pittsburgh) for supporting ongoing translation research in mesenchymal stem cells and wound healing and Dr. Charlene Chu for expertise on autophagy.

\section{References}

1. Nussbaum SR, Carter MJ, Fife CE, DaVanzo J, Haught R, Nusgart M, Cartwright D: An economic evaluation of the impact, cost, and Medicare policy implications of chronic nonhealing wounds. Value Health 2018, 21:27-32

2. Frykberg RG, Banks J: Challenges in the treatment of chronic wounds. Adv Wound Care (New Rochelle) 2015, 4:560-582

3. Yates CC, Bodnar R, Wells A: Matrix control of scarring. Cell Mol Life Sci 2011, 68:1871-1881

4. Lazarus GS, Cooper DM, Knighton DR, Percoraro RE, Rodeheaver G, Robson MC: Definitions and guidelines for assessment of wounds and evaluation of healing. Wound Repair Regen 1994, 2:165-170

5. Yates CC, Hebda P, Wells A: Skin wound healing and scarring: fetal wounds and regenerative restitution. Birth Defects Res C Embryo Today 2012, 96:325-333

6. Barker TH, Engler AJ: The provisional matrix: setting the stage for tissue repair outcomes. Matrix Biol 2017, 60-61:1-4

7. Bodnar RJ, Rodgers ME, Chen WC, Wells A: Pericyte regulation of vascular remodeling through the CXC receptor 3. Arterioscler Thromb Vasc Biol 2013, 33:2818-2829

8. Martins-Green M, Petreaca M, Wang L: Chemokines and their receptors are key players in the orchestra that regulates wound healing. Adv Wound Care 2013, 2:327-347

9. Sahota PS, Burn JL, Heaton M, Freedlander E, Suvarna SK, Brown NJ, Mac Neil S: Development of a reconstructed human skin model for angiogenesis. Wound Repair Regen 2003, 11:275-284

10. Benayahu D, Kletter Y, Zipori D, Wientroub S: Bone marrow-derived stromal cell line expressing osteoblastic phenotype in vitro and osteogenic capacity in vivo. J Cell Physiol 1989, 140:1-7

11. Bruder SP, Kurth AA, Shea M, Hayes WC, Jaiswal N, Kadiyala S: Bone regeneration by implantation of purified, culture-expanded human mesenchymal stem cells. J Orthop Res 1998, 16:155-162

12. Ferrari G, Cusella-De Angelis G, Coletta M, Paolucci E, Stornaiuolo A, Cossu G, Mavilio F: Muscle regeneration by bone marrow-derived myogenic progenitors. Science 1998, 279: $1528-1530$

13. Hauner H, Schmid P, Pfeiffer EF: Glucocorticoids and insulin promote the differentiation of human adipocyte precursor cells into fat cells. J Clin Endocrinol Metab 1987, 64:832-835

14. Johnstone B, Hering TM, Caplan AI, Goldberg VM, Yoo JU: In vitro chondrogenesis of bone marrow-derived mesenchymal progenitor cells. Exp Cell Res 1998, 238:265-272

15. Pittenger MF, Mackay AM, Beck SC, Jaiswal RK, Douglas R, Mosca JD, Moorman MA, Simonetti DW, Craig S, Marshak DR: Multilineage potential of adult human mesenchymal stem cells. Science 1999, 284:143-147

16. Wakitani S, Saito T, Caplan AI: Myogenic cells derived from rat bone marrow mesenchymal stem cells exposed to 5-azacytidine. Muscle Nerve 1995, 18:1417-1426

17. Viswanathan S, Shi Y, Galipeau J, Krampera M, Leblanc K, Martin I, Nolta J, Phinney DG, Sensebe L: Mesenchymal stem versus stromal cells: International Society for Cell \& Gene Therapy (ISCT(R)) Mesenchymal Stromal Cell Committee position statement on nomenclature. Cytotherapy 2019, 21:1019-1024

18. Singer NG, Caplan AI: Mesenchymal stem cells: mechanisms of inflammation. Annu Rev Pathol 2011, 6:457-478

19. Madrigal M, Rao KS, Riordan NH: A review of therapeutic effects of mesenchymal stem cell secretions and induction of secretory modification by different culture methods. J Transl Med 2014, 12: 260

20. Shin L, Peterson DA: Human mesenchymal stem cell grafts enhance normal and impaired wound healing by recruiting existing endogenous tissue stem/progenitor cells. Stem Cells Transl Med 2013, 2: $33-42$

21. Hu MS, Borrelli MR, Lorenz HP, Longaker MT, Wan DC: Mesenchymal stromal cells and cutaneous wound healing: a comprehensive review of the background, role, and therapeutic potential. Stem Cells Int 2018, 2018:6901983

22. Chen L, Tredget EE, Wu PY, Wu Y: Paracrine factors of mesenchymal stem cells recruit macrophages and endothelial lineage cells and enhance wound healing. PLoS One 2008, 3:e1886

23. Di Ianni M, Del Papa B, De Ioanni M, Moretti L, Bonifacio E, Cecchini D, Sportoletti P, Falzetti F, Tabilio A: Mesenchymal cells recruit and regulate $\mathrm{T}$ regulatory cells. Exp Hematol 2008, 36: 309-318

24. Vasandan AB, Jahnavi S, Shashank C, Prasad P, Kumar A, Prasanna SJ: Human mesenchymal stem cells program macrophage plasticity by altering their metabolic status via a PGE2-dependent mechanism. Sci Rep 2016, 6:38308

25. Joel MDM, Yuan J, Wang J, Yan Y, Qian H, Zhang X, Xu W, Mao F: MSC: immunoregulatory effects, roles on neutrophils and evolving clinical potentials. Am J Transl Res 2019, 11:3890-3904

26. Yates CC, Rodrigues M, Nuschke A, Johnson ZI, Whaley D, Stolz D, Newsome J, Wells A: Multipotent stromal cells/mesenchymal stem cells and fibroblasts combine to minimize skin hypertrophic scarring. Stem Cell Res Ther 2017, 8:193

27. Huang S, Wu Y, Gao D, Fu X: Paracrine action of mesenchymal stromal cells delivered by microspheres contributes to cutaneous wound healing and prevents scar formation in mice. Cytotherapy 2015, 17:922-931

28. Machado Cde V, Telles PD, Nascimento IL: Immunological characteristics of mesenchymal stem cells. Rev Bras Hematol Hemoter 2013, 35:62-67

29. Rodrigues M, Yates CC, Nuschke A, Griffith L, Wells A: The matrikine tenascin-C protects multipotential stromal cells/mesenchymal stem cells from death cytokines such as FasL. Tissue Eng Part A 2013, 19:1972-1983

30. Kornicka K, Houston J, Marycz K: Dysfunction of mesenchymal stem cells isolated from metabolic syndrome and type 2 diabetic patients as result of oxidative stress and autophagy may limit their potential therapeutic use. Stem Cell Rev Rep 2018, 14:337-345 
31. Pittenger MF, Martin BJ: Mesenchymal stem cells and their potential as cardiac therapeutics. Circ Res 2004, 95:9-20

32. Yang M, Wei $\mathrm{X}$, Li J, Heine LA, Rosenwasser R, Iacovitti L: Changes in host blood factors and brain glia accompanying the functional recovery after systemic administration of bone marrow stem cells in ischemic stroke rats. Cell Transplant 2010, 19: $1073-1084$

33. Sagrinati C, Ronconi E, Lazzeri E, Lasagni L, Romagnani P: Stemcell approaches for kidney repair: choosing the right cells. Trends Mol Med 2008, 14:277-285

34. Toma C, Pittenger MF, Cahill KS, Byrne BJ, Kessler PD: Human mesenchymal stem cells differentiate to a cardiomyocyte phenotype in the adult murine heart. Circulation 2002, 105:93-98

35. Yates CC, Nuschke A, Rodrigues M, Whaley D, Dechant JJ, Taylor DP, Wells A: Improved transplanted stem cell survival in a polymer gel supplemented with tenascin $\mathrm{C}$ accelerates healing and reduces scarring of murine skin wounds. Cell Transpl 2017, 26: $103-113$

36. Hu MS, Leavitt T, Malhotra S, Duscher D, Pollhammer MS, Walmsley GG, Maan ZN, Cheung AT, Schmidt M, Huemer GM, Longaker MT, Lorenz HP: Stem cell-based therapeutics to improve wound healing. Plast Surg Int 2015, 2015:383581

37. Rodrigues M, Griffith LG, Wells A: Growth factor regulation of proliferation and survival of multipotential stromal cells. Stem Cell Res Ther 2010, 1:32

38. Song H, Song BW, Cha MJ, Choi IG, Hwang KC: Modification of mesenchymal stem cells for cardiac regeneration. Expert Opin Biol Ther 2010, 10:309-319

39. Rodrigues M, Turner O, Stolz D, Griffith LG, Wells A: Production of reactive oxygen species by multipotent stromal cells/mesenchymal stem cells upon exposure to fas ligand. Cell Transpl 2012, 21: $2171-2187$

40. Nuschke A, Rodrigues M, Wells AW, Sylakowski K, Wells A: Mesenchymal stem cells/multipotent stromal cells (MSCs) are glycolytic and thus glucose is a limiting factor of in vitro models of MSC starvation. Stem Cell Res Ther 2016, 7:179

41. Zhu W, Chen J, Cong X, Hu S, Chen X: Hypoxia and serum deprivation-induced apoptosis in mesenchymal stem cells. Stem Cells 2006, 24:416-425

42. Potier E, Ferreira E, Meunier A, Sedel L, Logeart-Avramoglou D, Petite H: Prolonged hypoxia concomitant with serum deprivation induces massive human mesenchymal stem cell death. Tissue Eng 2007, 13:1325-1331

43. Marino G, Niso-Santano M, Baehrecke EH, Kroemer G: Self-consumption: the interplay of autophagy and apoptosis. Nat Rev Mol Cell Biol 2014, 15:81-94

44. Li L, Chen X, Wang WE, Zeng C: How to improve the survival of transplanted mesenchymal stem cell in ischemic heart? Stem Cells Int 2016, 2016:9682757

45. Otero-Vinas M, Falanga V: Mesenchymal stem cells in chronic wounds: the spectrum from basic to advanced therapy. Adv Wound Care (New Rochelle) 2016, 5:149-163

46. Somaiah C, Kumar A, Mawrie D, Sharma A, Patil SD, Bhattacharyya J, Swaminathan R, Jaganathan BG: Collagen promotes higher adhesion, survival and proliferation of mesenchymal stem cells. PLoS One 2015, 10:e0145068

47. Popov C, Radic T, Haasters F, Prall WC, Aszodi A, Gullberg D, Schieker M, Docheva D: Integrins alpha2beta1 and alpha1lbetal regulate the survival of mesenchymal stem cells on collagen I. Cell Death Dis 2011, 2:e186

48. Smeriglio P, Lee J, Bhutani N: Soluble collagen VI treatment enhances mesenchymal stem cells expansion for engineering cartilage. Bioeng Transl Med 2017, 2:278-284

49. Castagnaro S, Chrisam M, Cescon M, Braghetta P, Grumati P, Bonaldo P: Extracellular collagen VI has prosurvival and autophagy instructive properties in mouse fibroblasts. Front Physiol 2018, 9: 1129
50. Murphy-Ullrich JE, Sage EH: Revisiting the matricellular concept. Matrix Biol 2014, 37:1-14

51. Iyer AK, Tran KT, Borysenko $\mathrm{CW}$, Cascio $\mathrm{M}$, Camacho $\mathrm{CJ}$, Blair HC, Bahar I, Wells A: Tenascin cytotactin epidermal growth factor-like repeat binds epidermal growth factor receptor with low affinity. J Cell Physiol 2007, 211:748-758

52. Schenk S, Hintermann E, Bilban M, Koshikawa N, Hojilla C, Khokha R, Quaranta V: Binding to EGF receptor of a laminin-5 EGFlike fragment liberated during MMP-dependent mammary gland involution. J Cell Biol 2003, 161:197-209

53. Wang L, Wang F, Zhao L, Yang W, Wan X, Yue C, Mo Z: Mesenchymal stem cells coated by the extracellular matrix promote wound healing in diabetic rats. Stem Cells Int 2019, 2019:9564869

54. Abdeen AA, Weiss JB, Lee J, Kilian KA: Matrix composition and mechanics direct proangiogenic signaling from mesenchymal stem cells. Tissue Eng Part A 2014, 20:2737-2745

55. Karoubi G, Ormiston ML, Stewart DJ, Courtman DW: Single-cell hydrogel encapsulation for enhanced survival of human marrow stromal cells. Biomaterials 2009, 30:5445-5455

56. Margadant C, Raymond K, Kreft M, Sachs N, Janssen H, Sonnenberg A: Integrin alpha3betal inhibits directional migration and wound re-epithelialization in the skin. J Cell Sci 2009, 122: $278-288$

57. Agah A, Kyriakides TR, Lawler J, Bornstein P: The lack of thrombospondin-1 (TSP1) dictates the course of wound healing in double-TSP1/TSP2-null mice. Am J Pathol 2002, 161:831-839

58. Midwood KS, Chiquet M, Tucker RP, Orend G: Tenascin-C at a glance. J Cell Sci 2016, 129:4321-4327

59. Ragelle H, Naba A, Larson BL, Zhou F, Prijic M, Whittaker CA, Del Rosario A, Langer R, Hynes RO, Anderson DG: Comprehensive proteomic characterization of stem cell-derived extracellular matrices. Biomaterials 2017, 128:147-159

60. Midwood KS, Orend G: The role of tenascin-C in tissue injury and tumorigenesis. J Cell Commun Signal 2009, 3:287-310

61. Kimura W, Sadek HA: The cardiac hypoxic niche: emerging role of hypoxic microenvironment in cardiac progenitors. Cardiovasc Diagn Ther 2012, 2:278-289

62. Ivanovic Z: Hypoxia or in situ normoxia: the stem cell paradigm. J Cell Physiol 2009, 219:271-275

63. Rosova I, Dao M, Capoccia B, Link D, Nolta JA: Hypoxic preconditioning results in increased motility and improved therapeutic potential of human mesenchymal stem cells. Stem Cells 2008, 26: $2173-2182$

64. Ejtehadifar M, Shamsasenjan K, Movassaghpour A, Akbarzadehlaleh P, Dehdilani N, Abbasi P, Molaeipour Z, Saleh M: The effect of hypoxia on mesenchymal stem cell biology. Adv Pharm Bull 2015, 5:141-149

65. Basciano L, Nemos C, Foliguet B, de Isla N, de Carvalho M, Tran N, Dalloul A: Long term culture of mesenchymal stem cells in hypoxia promotes a genetic program maintaining their undifferentiated and multipotent status. BMC Cell Biol 2011, 12:12

66. Moya A, Paquet J, Deschepper M, Larochette N, Oudina K, Denoeud C, Bensidhoum M, Logeart-Avramoglou D, Petite H: Human mesenchymal stem cell failure to adapt to glucose shortage and rapidly use intracellular energy reserves through glycolysis explains poor cell survival after implantation. Stem Cells 2018, 36: 363-376

67. Palomaki S, Pietila M, Laitinen S, Pesala J, Sormunen R, Lehenkari P, Koivunen P: HIF-1alpha is upregulated in human mesenchymal stem cells. Stem Cells 2013, 31:1902-1909

68. Wagegg M, Gaber T, Lohanatha FL, Hahne M, Strehl C, Fangradt M, Tran CL, Schonbeck K, Hoff P, Ode A, Perka C, Duda GN, Buttgereit F: Hypoxia promotes osteogenesis but suppresses adipogenesis of human mesenchymal stromal cells in a hypoxia-inducible factor-1 dependent manner. PLoS One 2012, 7:e46483

69. Lv B, Li F, Fang J, Xu L, Sun C, Han J, Hua T, Zhang Z, Feng Z, Jiang X: Hypoxia inducible factor 1alpha promotes survival of 
mesenchymal stem cells under hypoxia. Am J Transl Res 2017, 9: $1521-1529$

70. Beegle J, Lakatos K, Kalomoiris S, Stewart H, Isseroff RR, Nolta JA, Fierro FA: Hypoxic preconditioning of mesenchymal stromal cells induces metabolic changes, enhances survival, and promotes cell retention in vivo. Stem Cells 2015, 33:1818-1828

71. Papandreou I, Cairns RA, Fontana L, Lim AL, Denko NC: HIF-1 mediates adaptation to hypoxia by actively downregulating mitochondrial oxygen consumption. Cell Metab 2006, 3:187-197

72. Lee JH, Yoon YM, Lee SH: Hypoxic preconditioning promotes the bioactivities of mesenchymal stem cells via the HIF-1alpha-GRP78Akt axis. Int J Mol Sci 2017, 18:1320

73. Saini U, Gumina RJ, Wolfe B, Kuppusamy ML, Kuppusamy P, Boudoulas KD: Preconditioning mesenchymal stem cells with caspase inhibition and hyperoxia prior to hypoxia exposure increases cell proliferation. J Cell Biochem 2013, 114:2612-2623

74. Mazure NM, Pouyssegur J: Hypoxia-induced autophagy: cell death or cell survival? Curr Opin Cell Biol 2010, 22:177-180

75. Yang Z, Klionsky DJ: Mammalian autophagy: core molecular machinery and signaling regulation. Curr Opin Cell Biol 2010, 22: 124-131

76. Klionsky DJ, Cuervo AM, Dunn WA Jr, Levine B, van der Klei I, Seglen PO: How shall I eat thee? Autophagy 2007, 3:413-416

77. Mizushima N, Levine B, Cuervo AM, Klionsky DJ: Autophagy fights disease through cellular self-digestion. Nature 2008, 451:1069-1075

78. Levine B, Yuan J: Autophagy in cell death: an innocent convict? J Clin Invest 2005, 115:2679-2688

79. Maiuri MC, Zalckvar E, Kimchi A, Kroemer G: Self-eating and selfkilling: crosstalk between autophagy and apoptosis. Nat Rev Mol Cell Biol 2007, 8:741-752

80. Hu C, Zhao L, Wu D, Li L: Modulating autophagy in mesenchymal stem cells effectively protects against hypoxia- or ischemia-induced injury. Stem Cell Res Ther 2019, 10:120

81. Oliver L, Hue E, Priault M, Vallette FM: Basal autophagy decreased during the differentiation of human adult mesenchymal stem cells. Stem Cells Dev 2012, 21:2779-2788

82. Lee Y, Jung J, Cho KJ, Lee SK, Park JW, Oh IH, Kim GJ: Increased SCF/c-kit by hypoxia promotes autophagy of human placental chorionic plate-derived mesenchymal stem cells via regulating the phosphorylation of mTOR. J Cell Biochem 2013, 114:79-88

83. Nuschke A, Rodrigues M, Stolz DB, Chu CT, Griffith L, Wells A: Human mesenchymal stem cells/multipotent stromal cells consume accumulated autophagosomes early in differentiation. Stem Cell Res Ther 2014, 5:140

84. Pantovic A, Krstic A, Janjetovic K, Kocic J, Harhaji-Trajkovic L, Bugarski D, Trajkovic V: Coordinated time-dependent modulation of AMPK/Akt/mTOR signaling and autophagy controls osteogenic differentiation of human mesenchymal stem cells. Bone 2013, 52: $524-531$

85. Bellot G, Garcia-Medina R, Gounon P, Chiche J, Roux D, Pouyssegur J, Mazure NM: Hypoxia-induced autophagy is mediated through hypoxia-inducible factor induction of BNIP3 and BNIP3L via their BH3 domains. Mol Cell Biol 2009, 29:2570-2581

86. Janji B, Viry E, Moussay E, Paggetti J, Arakelian T, Mgrditchian T, Messai Y, Noman MZ, Van Moer K, Hasmim M, Mami-Chouaib F, Berchem G, Chouaib S: The multifaceted role of autophagy in tumor evasion from immune surveillance. Oncotarget 2016, 7: $17591-17607$

87. An Y, Liu WJ, Xue P, Ma Y, Zhang LQ, Zhu B, Qi M, Li LY, Zhang YJ, Wang QT, Jin Y: Autophagy promotes MSC-mediated vascularization in cutaneous wound healing via regulation of VEGF secretion. Cell Death Dis 2018, 9:58

88. Liu J, Hao H, Huang H, Tong C, Ti D, Dong L, Chen D, Zhao Y, Liu H, Han W, Fu X: Hypoxia regulates the therapeutic potential of mesenchymal stem cells through enhanced autophagy. Int J Low Extrem Wounds 2015, 14:63-72

89. Zhang Z, Yang C, Shen M, Yang M, Jin Z, Ding L, Jiang W, Yang J, Chen H, Cao F, Hu T: Autophagy mediates the beneficial effect of hypoxic preconditioning on bone marrow mesenchymal stem cells for the therapy of myocardial infarction. Stem Cell Res Ther 2017, 8:89

90. Neill T, Schaefer L, Iozzo RV: Instructive roles of extracellular matrix on autophagy. Am J Pathol 2014, 184:2146-2153

91. Gubbiotti MA, Buraschi S, Kapoor A, Iozzo RV: Proteoglycan signaling in tumor angiogenesis and endothelial cell autophagy. Semin Cancer Biol 2020, 62:1-8

92. Buraschi S, Neill T, Iozzo RV: Decorin is a devouring proteoglycan: remodeling of intracellular catabolism via autophagy and mitophagy. Matrix Biol 2019, 75-76:260-270

93. Wei Y, Zou Z, Becker N, Anderson M, Sumpter R, Xiao G, Kinch L, Koduru P, Christudass CS, Veltri RW, Grishin NV, Peyton M, Minna J, Bhagat G, Levine B: EGFR-mediated Beclin 1 phosphorylation in autophagy suppression, tumor progression, and tumor chemoresistance. Cell 2013, 154:1269-1284

94. Wang B, Ling S, Lin WC: 14-3-3Tau regulates Beclin 1 and is required for autophagy. PLoS One 2010, 5:e10409

95. Fung C, Lock R, Gao S, Salas E, Debnath J: Induction of autophagy during extracellular matrix detachment promotes cell survival. Mol Biol Cell 2008, 19:797-806

96. Xu Q, Liu W, Liu X, Otkur W, Hayashi T, Yamato M, Fujisaki H, Hattori S, Tashiro SI, Ikejima T: Type I collagen promotes primary cilia growth through down-regulating HDAC6-mediated autophagy in confluent mouse embryo fibroblast 3T3-L1 cells. J Biosci Bioeng 2018, 125:8-14 